\title{
Transient Response of the Acid-base Diode for Polarity Change
}

\author{
Viktória Koncz ${ }^{1 *}$, Zoltán Noszticzius ${ }^{1}$ Kristóf Kály-Kullai \\ 1 Department of Physics, Faculty of Natural Sciences, Budapest University of Technology and Economics, H-1521 Budapest, \\ P. O. B. 91, Hungary \\ * Corresponding author, e-mail: viki.koncz@gmail.com
}

Received: 10 January 2021, Accepted: 12 May 2021, Published online: 18 August 2021

\begin{abstract}
An application of the so-called acid-base diode would be the sensitive detection of nonhydrogen cations in an acidic medium based on salt-effects. For diode purposes different connecting elements between the acidic and aqueous reservoirs of the diode were developed, namely a polyvinyl alcohol (PVA) hydrogel cylinder, and a polyvinyl butyral (PVB) membrane. During the measurement of the voltage - current characteristic (VCC) of the diode, it was found, that in the case of PVA gel cylinder an overshoot (a local maximum followed by a local minimum) appeared in the time vs. current curve, while the diode was switched between modes (open or closed), that is the direction of the applied voltage was reversed. The overshoot did not appear in PVB membrane.

The existence of overshoots was studied by numerical simulations. The time response of the diode with different hypothetic connecting elements was investigated, when the diode was switched between modes via changing the polarity of applied voltage. We found that larger diffusion coefficients of hydrogen and hydroxide ions explain the appearance of overshoots. By examining the concentration and potential profiles a qualitative explanation of this phenomenon was given.
\end{abstract}

\section{Keywords}

acid-base diodes, FEM modeling, COMSOL Multiphysics

\section{Introduction}

\subsection{Concept of an acid-base diode}

An acid-base diode (electrolyte diode) is a reaction-diffusion-electric migration system [1-3]. Main parts of an acid-base diode are shown in Fig. 1 [4]: an alkaline (e.g.

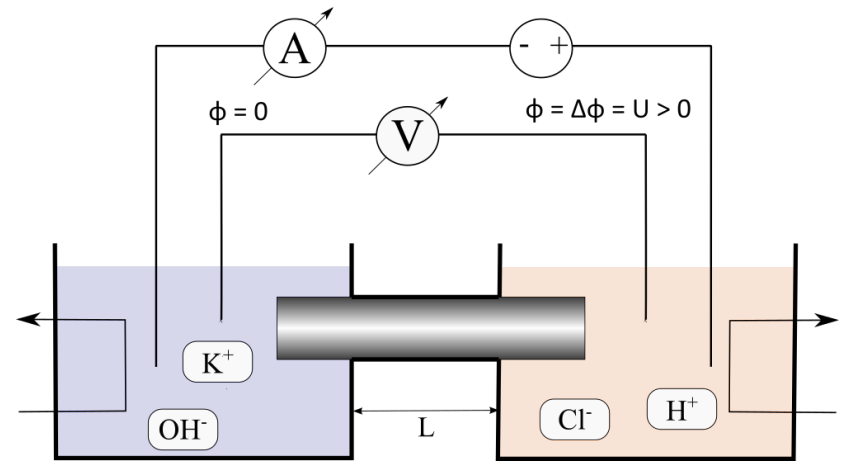

Fig. 1 Schematic view of a strong acid-base diode: an acidic reservoir (containing e.g. $0.1 \mathrm{M} \mathrm{HCl}$ solution) is attached to an alkaline one (containing e.g. $0.1 \mathrm{M} \mathrm{KOH}$ solution) via a connecting element (hydrogel or membrane). If $\Delta \phi>0$ the diode is reverse biased, otherwise it is forward biased. This figure is mainly the reproduction of our previously published figure [4].
$0.1 \mathrm{M}$ aqueous $\mathrm{KOH}$ ) and an acidic (e. g. 0.1 M aqueous $\mathrm{HCl}$ ) reservoirs are connected by a hydrogel or membrane (connecting element). Constant and homogeneous electrolyte concentrations are maintained in the reservoirs (both are continuously fed by fresh solutions), while the connecting element is an unstirred zone. Within the connecting element diffusion, ionic migration and chemical reactions are allowed, it restrains any advection or convection.

The voltage - current characteristic (VCC) of the diode obtained by applying electric potential between the reservoirs is similar to the characteristic of a semiconductor diode (see Fig. 2) [4, 5]. In the forward biased direction potassium $\left(\mathrm{K}^{+}\right)$and chloride $\left(\mathrm{Cl}^{-}\right)$ions migrate into the connecting element, where a well-conducting salt (in this case $\mathrm{KCl}$ ) is formed. On the other hand, in the case of opposite polarity the electric field pulls hydrogen and hydroxide ions into the connecting element, where they react and form water.

During the previously carried out experiments two types of connecting elements were used for diode purposes: 


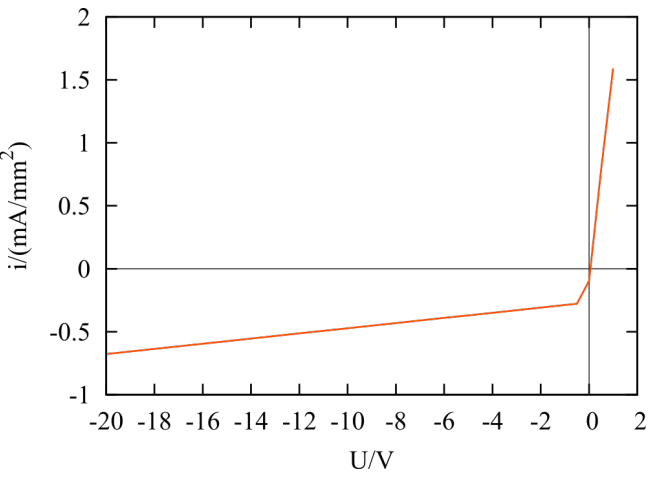

Fig. 2 Calculated voltage - current density characteristic of a strong acid-base diode. The characteristic of the diode was previously published $[4,5]$.

a PVA-based gel cylinder, and a PVB membrane. The PVA gel contains fixed weakly acidic groups, their dissociation leads to fixed negative ions $[6,7]$. $\left(\mathrm{FA}^{-}\right.$denotes the fixed charge, and HFA is its protonated form.)

\subsection{Motivation of the research}

Due to the slow time response of the diode when PVA gel cylinder is used as a connecting element, a much thinner (and faster) PVB membrane was developed [8]. It was observed that during periodic switching of voltage between forward and reverse modes, an overshoot (a local maximum followed by a local minimum) appears in the measured current, when PVA gel cylinder is used as connecting element. A similar transient response of the diode was observed with PVA hydrogel in $[9,10]$. This overshoot is missing, if PVB membrane is applied instead [7, 8]. For more details about these earlier experimental results see the Appendix.

We hypothesize that this difference in the characteristics observed in different gels is primarily caused by different diffusion of $\mathrm{H}^{+}$and $\mathrm{OH}^{-}$ions in those media. The PVA hydrogel is assumed to be an aqueous medium, thus due to the Grotthus mechanism the hydrogen and hydroxyl ions have unusually high diffusion coefficients compared to other ions (like $\mathrm{K}^{+}$and $\mathrm{Cl}^{-}$), causing increased mobilities. In the PVB membrane there is no Grotthus mechanism, because it is a non-aqueous medium. Thus, diffusion coefficients of $\mathrm{H}^{+}$and $\mathrm{OH}^{-}$are roughly equal to that of $\mathrm{K}^{+}$and $\mathrm{Cl}^{-}$.

The aim of this research is to investigate the time response of the diode for voltage modification if the diode is switched from closed to open mode in different types of hypothetic connecting elements via model calculations. In the simulation study the diffusion coefficient of hydrogen and hydroxyl ions is altered.
We would like to mention, that during the last decade far fewer publications were written about this topic, than in previous years. The cited publications contain all relevant historical information, on which the current investigation is based.

\section{Methods}

\subsection{The model}

The mathematical model of the acid-base diode is the same as previously used $[4,5]$. A system of one-dimensional partial differential equations describes the behavior of the diode [11-14], during a model calculation this is solved with different boundary conditions. The previously described simplifications and assumptions are kept (one dimensional model, concentration polarization and ohmic potential drop on the reservoirs are neglected) $[5,6]$.

The concentration profile of the $i$-th component can be calculated from the mass-balance equation $[15,16]$, in which the total flux of the $i$-th component is obtained from the Nernst-Planck equation:

$\partial c_{i} / \partial t=-\partial / \partial x\left(-D_{i} \partial c_{i} / \partial x-c_{i} z_{i} D_{i} F / R T \partial \phi / \partial x\right)+\sigma_{i}$,

where $D_{i}, c_{i}$ and $z_{i}$ denote the diffusion coefficient, concentration and charge number of the $i$-th component $\left(\mathrm{H}^{+}, \mathrm{OH}^{-}\right.$, $\mathrm{K}^{+}, \mathrm{Cl}^{-}, \mathrm{FA}^{-}, \mathrm{HFA}$ ), respectively. $R$ is the molar gas constant, $F$ is the Faraday constant, $T$ is the temperature, $\Phi$ is the space dependent potential and $x$ denotes the space coordinate along the gel cylinder. $\sigma_{i}$ denotes the reaction rate (accumulation and consumption) of the $i$-th component:

$$
\begin{aligned}
& \sigma_{\mathrm{H}^{+}}=k_{w}\left(K_{w}-c_{\mathrm{H}^{+}} c_{\mathrm{OH}^{-}}\right)+k_{f}\left(K_{f} c_{\mathrm{HFA}}-c_{\mathrm{H}^{+}} c_{\mathrm{FA}^{-}}\right), \\
& \sigma_{\mathrm{OH}^{-}}=k_{w}\left(K_{w}-c_{\mathrm{H}^{+}} c_{\mathrm{OH}^{-}}\right), \\
& \sigma_{\mathrm{K}^{+}}=\sigma_{\mathrm{Cl}^{-}}=0, \\
& \sigma_{\mathrm{FA}^{-}}=-\sigma_{\mathrm{HFA}}=k_{f}\left(K_{f} c_{\mathrm{HFA}}-c_{\mathrm{H}^{+}} c_{\mathrm{FA}^{-}}\right),
\end{aligned}
$$

in which $k_{w}$ is the rate constant of water recombination, and $K_{w}$ is the equilibrium constant of water dissociation. $k_{f}$ denotes the kinetic constant and $K_{f}$ is the dissociation constant of the fixed charge.

The potential profile of the diode is calculated from the Poisson equation

$\partial / \partial x(-\varepsilon \partial \phi / \partial x)=F\left(\sum z_{i} c_{i}\right)$,

where $\varepsilon$ denotes the permittivity of water.

For stationary investigations the concentration of the fixed anions can be calculated from the equilibrium, 
$c_{\mathrm{FA}^{-}}=c_{f} /\left(c_{\mathrm{H}^{+}} / K_{f}+1\right)$,

where $c_{f}$ denotes the total concentration of the fixed groups.

Therefore, the mass balance equation must be set only for mobile species. This assumption of quasi-stationarity was considered to be valid and was applied to solve the time-dependent model. The applied values of the parameters are found in Table 1 [17].

\subsection{Solution method}

The simulation framework was developed in Java using COMSOL Multiphysics (version 4.3) software package,

Table 1 Model parameters [17]

\begin{tabular}{|c|c|c|}
\hline Parameter (unit) & Description & Value \\
\hline$c_{\mathrm{KOH}}\left(\mathrm{mol} \mathrm{dm}^{-3}\right)$ & Concentration of base & 0.1 \\
\hline$c_{\mathrm{HCl}}\left(\mathrm{mol} \mathrm{dm}^{-3}\right)$ & Concentration of acid & 0.1 \\
\hline$D_{\mathrm{H}^{+}, a q}\left(\mathrm{~m}^{2} \mathrm{~s}^{-1}\right)$ & $\begin{array}{l}\text { Diffusion coeff. of protons } \\
\text { in water }\end{array}$ & $9.31 \times 10^{-9}$ \\
\hline$D_{\mathrm{OH}^{+}, a q}\left(\mathrm{~m}^{2} \mathrm{~s}^{-1}\right)$ & $\begin{array}{l}\text { Diffusion coeff. of hydroxyl } \\
\text { ions in water }\end{array}$ & $5.28 \times 10^{-9}$ \\
\hline$D_{K^{+}}\left(\mathrm{m}^{2} \mathrm{~s}^{-1}\right)$ & $\begin{array}{c}\text { Diffusion coeff. of potassium } \\
\text { ions }\end{array}$ & $1.96 \times 10^{-9}$ \\
\hline$D_{\mathrm{Cl}^{-}}\left(\mathrm{m}^{2} \mathrm{~s}^{-1}\right)$ & $\begin{array}{l}\text { Diffusion coeff. of chloride } \\
\text { ions }\end{array}$ & $2.04 \times 10^{-9}$ \\
\hline$D_{\mathrm{FA}^{-}}\left(\mathrm{m}^{2} \mathrm{~s}^{-1}\right)$ & $\begin{array}{c}\text { Diffusion coeff. of the fixed } \\
\text { charge }\end{array}$ & 0 \\
\hline$D_{\mathrm{HFA}}\left(\mathrm{m}^{2} \mathrm{~s}^{-1}\right)$ & $\begin{array}{l}\text { Diffusion coeff. of the } \\
\text { protonated fixed group }\end{array}$ & 0 \\
\hline$l(\mathrm{~m})$ & Length of the gel & $1 \times 10^{-3}$ \\
\hline$z_{\mathrm{H}^{+}}$ & Charge number of protons & 1 \\
\hline$z_{\mathrm{OH}^{-}}$ & $\begin{array}{c}\text { Charge number of hydroxyl } \\
\text { ions }\end{array}$ & -1 \\
\hline$z_{\mathrm{K}^{+}}$ & $\begin{array}{c}\text { Charge number of potassium } \\
\text { ions }\end{array}$ & 1 \\
\hline$z_{\mathrm{Cl}^{-}}$ & $\begin{array}{l}\text { Charge number of chloride } \\
\text { ions }\end{array}$ & -1 \\
\hline$z_{\mathrm{FA}^{-}}$ & $\begin{array}{c}\text { Charge number of the fixed } \\
\text { charge }\end{array}$ & -1 \\
\hline$z_{\mathrm{HFA}}$ & $\begin{array}{l}\text { Charge number of the } \\
\text { protonated fixed group }\end{array}$ & 0 \\
\hline$F\left(\mathrm{Cmol}^{-1}\right)$ & Faraday constant & $9.6487 \times 10^{4}$ \\
\hline$R\left(\mathrm{~J} \mathrm{~mol}^{-1} \mathrm{~K}^{-1}\right)$ & Molar gas constant & 8.314 \\
\hline$T(\mathrm{~K})$ & Temperature & 298.15 \\
\hline$\varepsilon\left(\mathrm{A} \mathrm{s} \mathrm{V}^{-1} \mathrm{~m}^{-1}\right)$ & Permittivity of water & $6.954 \times 10^{-10}$ \\
\hline$k_{w}\left(\mathrm{~m}^{3} \mathrm{~mol}^{-1} \mathrm{~s}^{-1}\right)$ & $\begin{array}{l}\text { Kinetic constant of water } \\
\text { recombination }\end{array}$ & $1.3 \times 10^{8}$ \\
\hline$K_{w}\left(\mathrm{~mol}^{2} \mathrm{~m}^{-6}\right)$ & Ionic product of water & $1 \times 10^{-8}$ \\
\hline$c_{f}\left(\mathrm{~mol} \mathrm{~m}^{-3}\right)$ & $\begin{array}{l}\text { Concentration of the fixed } \\
\text { charge }\end{array}$ & 4 \\
\hline$k_{f}\left(\mathrm{~m}^{3} \mathrm{~mol}^{-1} \mathrm{~s}^{-1}\right)$ & $\begin{array}{l}\text { Kinetic constant of the fixed } \\
\text { charge }\end{array}$ & $6 \times 10^{6}$ \\
\hline$K_{f}\left(\mathrm{~mol} \mathrm{~m}^{-3}\right)$ & $\begin{array}{l}\text { Dissociation constant of the } \\
\text { fixed charge }\end{array}$ & $1 \times 10^{-1}$ \\
\hline
\end{tabular}

which provides the $\mathrm{FEM}^{1}$ algorithms. To define the model the "Transport of Diluted Species" (chds) interface and the predefined "Classical PDEs/Poisson equation" interface were used [4].

After the calculation of the boundary conditions (Dirichlet boundary conditions regarding the Donnan equilibrium), the model was solved for stationary state to get the initial conditions. The time-dependent study is performed with a time-dependent boundary condition. In COMSOL GUI the time-dependent boundary condition is defined with a STEP function under the Model/ Definition node (see Fig. 3), where the value of the transition time $\left(t_{t r}\right)$ can also be set.

For the time-marching the BDF method with the SPOOLES linear solver (crosswind diffusion stabilization technique, automatic scaling) was used. The time interval of the time-dependent simulations was $400 \mathrm{~s}$ to achieve the new stationary state. The absolute tolerance value was set to $A_{\text {tol }}=10^{-5}$.

The mesh usually contained 30000 second order Lagrangian elements, the wide environment of the reaction zone (including all places where it can possibly appear) was covered by much finer elements, than other parts of the gel cylinder.

During a model calculation space and time dependent current densities are calculated along the gel cylinder. Further investigations are required to figure out, how the calculated current densities are related to the measured diode current in a real device. For now, the space average of current density along the gel cylinder is used, as it provides a quantity depending only on time, and showing qualitatively similar behavior than the current in a real diode.

\section{Results and discussion}

Our work is presented in three subsections. First, the transient response of the diode was investigated for voltage modification from $10 \mathrm{~V}$ to $-2 \mathrm{~V}$, which switches the diode from closed to open mode. After that, the influence of transition time $\left(t_{t r}\right)$ was studied, it was changed between le-7 s and $2 \mathrm{~s}$. In the case of small transition times the effects are more pronounced and easier to understand, for this reason we examined such small and experimentally hard to realise $t_{t r}$ values. Finally, the influence of smaller diffusion coefficients of $\mathrm{H}^{+}$ and $\mathrm{OH}^{-}$on the transient response of the diode was studied.

1 Finite element method is a variational method to approximate the solution of the discretized model by minimizing an associated error function. 


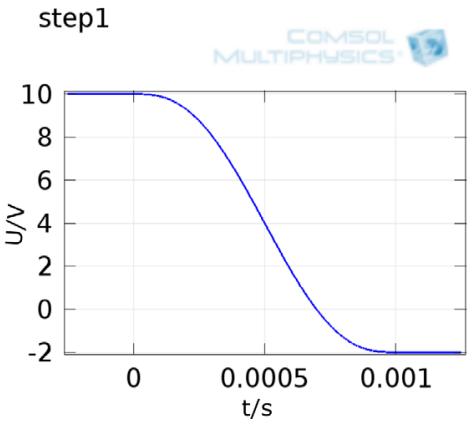

Fig. 3 The time-dependent boundary condition is defined with a STEP function in COMSOL. In the depicted case the size of the transition zone is $t_{t r}=0.001 \mathrm{~s}$, the location is at $t=0.0005 \mathrm{~s}$, the initial potential value is $10 \mathrm{~V}$ (corresponding to the initial stationary state, closed mode), and its final value is $-2 \mathrm{~V}$ (open mode).

\subsection{Transient response of the diode in PVA hydrogel}

The time-dependence of current density is shown in Fig. 4, while the diode is switched from closed to open mode. The time - current density curve depends on $t_{t r}$ significantly only in the first few seconds, after that the curves are basically the same. In this subsection we concentrate on the common part.

In the initial stationary state (closed mode) there are three distinguishable zones in the gel cylinder: an alkaline zone next to the alkaline reservoir, an acidic zone next to the acidic one, and between them a depletion zone, which is weakly acidic due to the presence of fixed charges. Because of the small amount of ions in the depletion zone, it has high ohmic resistance.

If the diode is switched from closed to open mode, as the result of the electric field's polarity change the $\mathrm{H}^{+}$ and $\mathrm{OH}^{-}$ions move out from the gel cylinder, simultaneously $\mathrm{K}^{+}$and $\mathrm{Cl}^{-}$ions enter it, and the three zones merge.

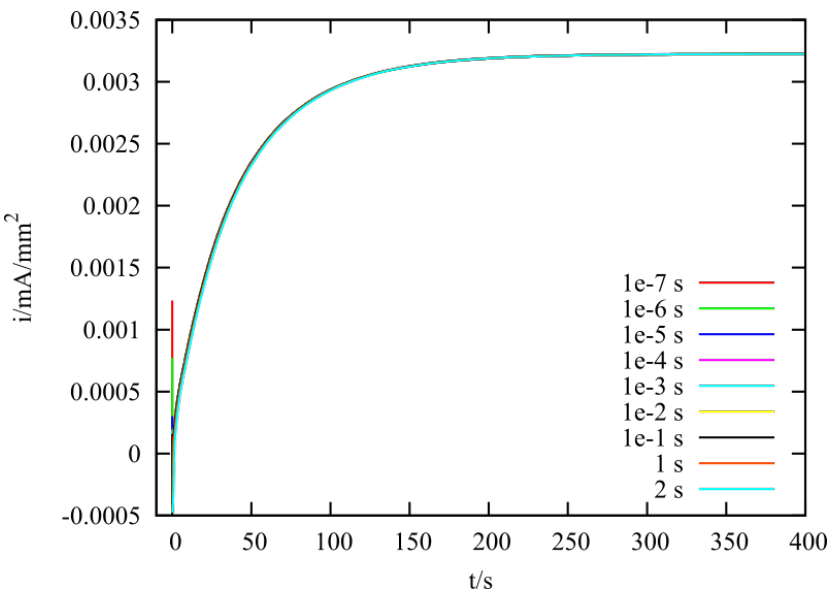

Fig. 4 Simulated time - current density curve of the diode in case of different $t_{t r}$ values, if the diode is switched from closed $(U=10 \mathrm{~V})$ to open mode $(U=-2 \mathrm{~V})$.
Approaching the new stationary state, the whole gel cylinder gets filled by $\mathrm{K}^{+}$and $\mathrm{Cl}^{-}$ions forming a well-conducting salt, which leads to a linear potential profile and high diode current. For the profiles see Figs. 5 and 6.

\subsection{The alteration of transition time}

If the transition time is small enough, an overshoot (a local maximum followed by a local minimum) appears in the first few seconds (see Fig. 7). Its size depends on $t_{t r}$, the smaller it is, the higher the overshoot becomes. In the case of $t_{t r}=2 \mathrm{~s}$, no local maximum is observable, the diode current increases monotonically. In our study the $t_{t r}$ is altered between $1 \mathrm{e}-7 \mathrm{~s}$ to $2 \mathrm{~s}$.

For a qualitative explanation of this overshoot it is easiest to consider the case of $t_{t r}=1 \mathrm{e}-7 \mathrm{~s}$. This very short period of time is not enough to move the ions (for the profiles see Fig. 8), thus the concentration profiles at $t=1 \mathrm{e}-7 \mathrm{~s}$ are still the same as in the initial steady state $(t=0 \mathrm{~s})$. The applied voltage has however completely changed. In the initial stationary state, most of the applied potential
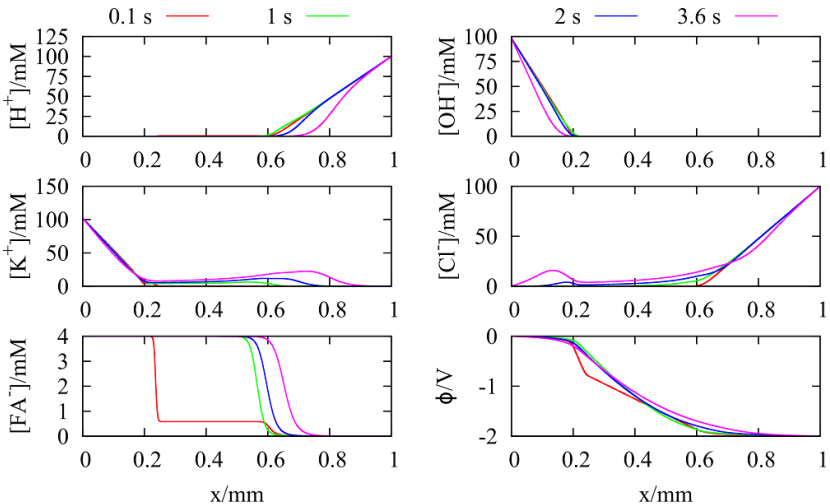

Fig. 5 Simulated concentration and potential profiles of the diode, if it is switched from closed $(U=10 \mathrm{~V})$ to open mode $(U=-2 \mathrm{~V}), t_{t r}=0.1 \mathrm{~s}$. Part I.
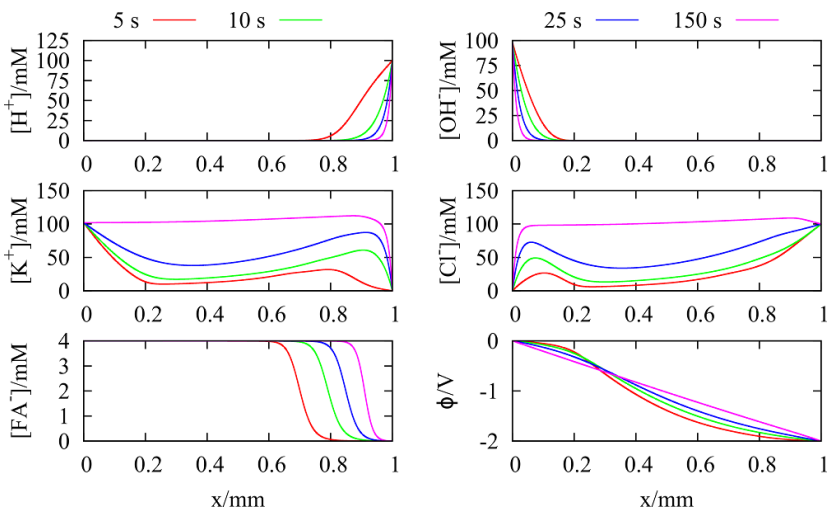

Fig. 6 Simulated concentration and potential profiles of the diode, if it is switched from closed $(U=10 \mathrm{~V})$ to open mode $(U=-2 \mathrm{~V}), t_{t r}=0.1 \mathrm{~s}$. Part II. 

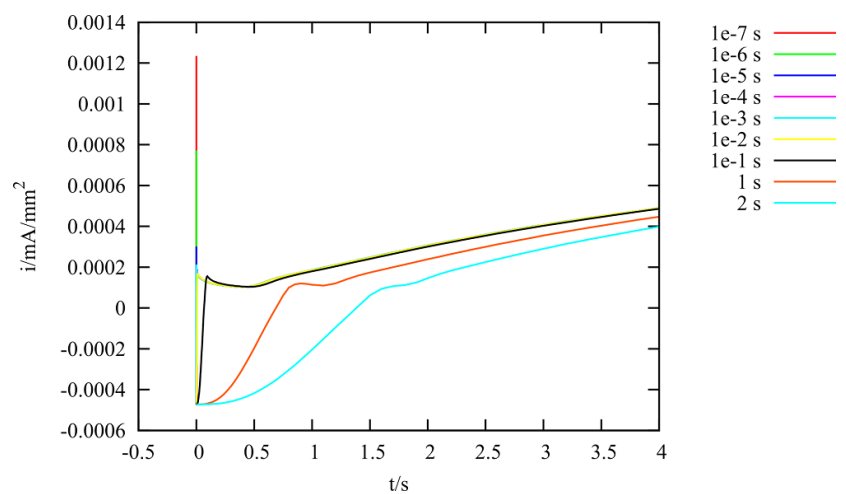

Fig. 7 Simulated time - current density curve of the diode in case of different $t_{t r}$ values, if the diode is switched from closed $(U=10 \mathrm{~V})$ to open mode $(U=-2 \mathrm{~V})$, Fig. 4 magnified between $t=0$ to $t=4 \mathrm{~s}$.
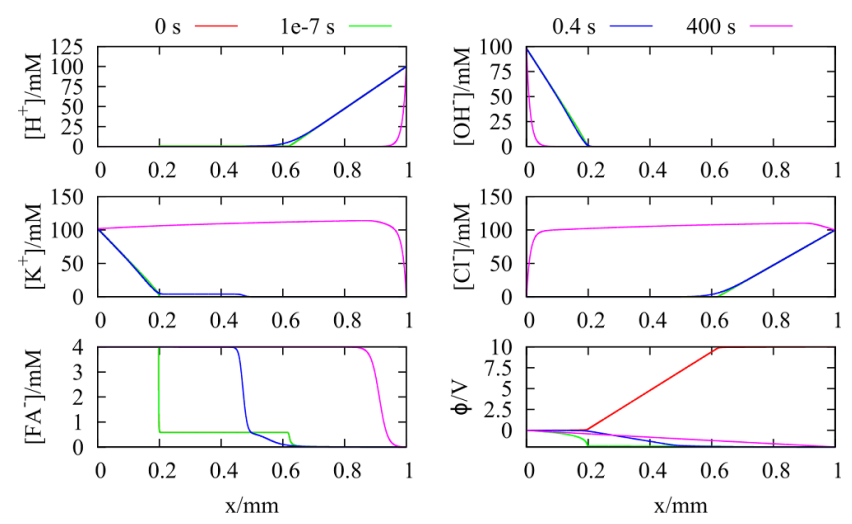

Fig. 8 Simulated concentration and potential profiles of the diode, if it is switched from closed $(U=10 \mathrm{~V})$ to open mode $(U=-2 \mathrm{~V})$, $t_{t r}=1 \mathrm{e}-7 \mathrm{~s} . t=1 \mathrm{e}-7 \mathrm{~s}$ belongs to the local maximum (overshoot) of the time - current density curve, and $t=0.4 \mathrm{~s}$ to the local minimum after the overshoot, respectively. Please note, that the concentration profiles in case of $t=0$ and $t=1 \mathrm{e}-7 \mathrm{~s}$ are very similar.

drops on the depletion zone. It results in a mostly negatively charged region between the alkaline and depletion zones, and in a positively charged one on the border of the acidic and the depletion zones. These charged regions remain almost intact at $t=1 \mathrm{e}-7 \mathrm{~s}$, when the applied voltage has completely changed. They distort the potential profile in such a way, that significant amount of the applied voltage drops on the well-conducting alkaline zone. This causes an increased current density corresponding to the local maximum. The interesting point is, that the absolute value of current density at $t=1 \mathrm{e}-7 \mathrm{~s}(U=-2 \mathrm{~V})$ is even higher than in the initial state $(U=10 \mathrm{~V})$.

The resulting currents decrease the charge density of these regions, causing more of the potential to drop on the depletion zone leading to decreased current. On the other hand, the depletion zone shrinks, and well-conducting ions slowly fill the gel. These opposing effects cause a local minimum (around $t=0.4 \mathrm{~s}$ ) in the current density curve. After that the concentration of $\mathrm{K}^{+}$and $\mathrm{Cl}^{-}$increases further in the gel cylinder (see Subsection 3.1).

Increasing the $t_{t r}$ time, during the voltage change the concentrations can already change somewhat. As a result, the potential drop on the alkaline zone is smaller due to already decreased charge densities at the borders. Thus, the local maximum occurs later, and its current decreases as well. If $t_{t r}$ is further increased, the overshoot even disappears. For the potential profiles in the cases of different $t_{t r}$ see Fig. 9.

\subsection{Reducing the diffusion coefficients of $\mathrm{H}^{+}$and $\mathrm{OH}^{-}$}

The PVA gel cylinder, which is a hydrogel, can be approximated by an aqueous medium regarding transport properties. For this reason, in the model calculations the aqueous diffusion coefficients of $\mathrm{H}^{+}$and $\mathrm{OH}^{-}$ions are used. The measurement of the voltage - current characteristic of the diode with PVB membrane suggested [8], that this approximation is not applicable to the PVB membrane. In [8] it was measured that $\left(D_{\mathrm{H}^{+}}+D_{\mathrm{OH}^{-}}\right)$is about 3-3.5 times bigger in PVA than in PVB because of the different mechanism of diffusion.

Let us introduce a new variable $p$, with which the aqueous diffusion coefficients of $\mathrm{H}^{+}$and $\mathrm{OH}^{-}$can be divided leading to diffusion coefficients in a hypothetic medium. It is assumed, that modifying the medium reduces the diffusion coefficients of $\mathrm{H}^{+}$and $\mathrm{OH}^{-}$with the same ratio:

$D_{\mathrm{H}^{+}}=D_{\mathrm{H}^{+}, a q} / p$,

$D_{\mathrm{OH}^{-}}=D_{\mathrm{OH}^{-}, a q} / p$.

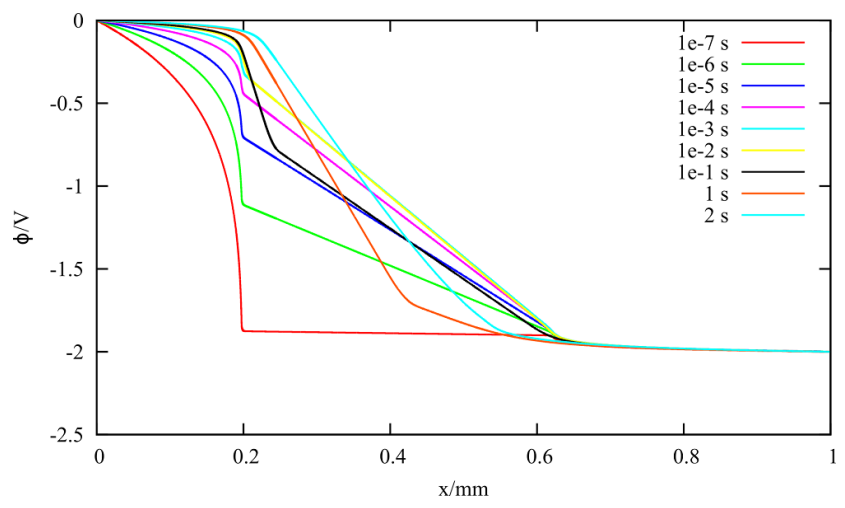

Fig. 9 Simulated potential profiles belonging to the overshoot of the diode in case of different $t_{t r}$ values. (In the case of $t_{t r}=2 \mathrm{~s}$, where no local maximum is observable, the depicted potential profile was chosen from $t=2 \mathrm{~s}$.) 
In this simulation study, the $p$ value was varied between 1 and 3, and with these hypothetic connecting elements the response of the diode for voltage change was studied. The case of $p=1$ corresponds to an aqueous medium like PVA, and $p=3$ was used to estimate the PVB membrane. In the case of $t_{t r}=0.1 \mathrm{~s}$ the time - current density curves for various $p$ are shown in Fig. 10. The bigger the $p$ value is, the smaller transition time is required for the overshoot to disappear $\left(t_{t r, \text { crit }}\right)$. (If $p=1, t_{t r, \text { crit }}$ is between 1 and $2 \mathrm{~s}$, if $p=1.5 t_{t r \text { crit }}$ is between $0.1 \mathrm{~s}$ and $1 \mathrm{~s}$ and in the cases of $p=2$ and $p=3 t_{t r, c r i t}$ is between $0.01 \mathrm{~s}$ and $0.1 \mathrm{~s}$.)

If $t_{t r}$ is small enough, there is overshoot even with $p=3$, but its amplitude decreases as $p$ increases (Fig. 11). It has two different causes. One, smaller diffusion coefficients result in smaller ion mobilities, which decreases the current. Two, this effect is further enhanced by different potential profiles having smaller potential drop in the well-conducting region. In case of $t_{t r}=1 \mathrm{e}-7 \mathrm{~s}$ the potential profiles for different $p$ values are shown in Fig. 12. If the diffusion coefficients of $\mathrm{H}^{+}$and $\mathrm{OH}^{-}$differ significantly from that of $\mathrm{K}^{+}$and $\mathrm{Cl}^{-}(p=1)$, due to the complex interaction of reaction, diffusion, and electric migration the local charge density becomes significantly larger at $t=1 \mathrm{e}-7 \mathrm{~s}$, than in the initial state. In the case of $p=3$ the local charge density remains similar to the initial state at $t=1 \mathrm{e}-7 \mathrm{~s}$ (corresponding to the local maximum). Because of the smaller local charge density, the potential distribution between the zones is more even, which certainly means less potential drop on the well-conducting region.

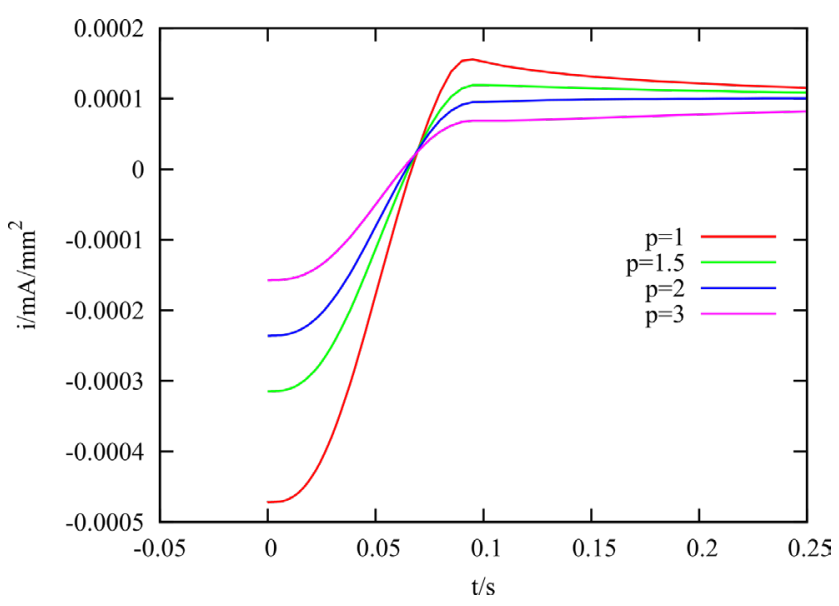

Fig. 10 Simulated time - current density curve of the diode (magnified between $t=0$ to $t=4 \mathrm{~s}$ ) in case of different $p$ values, if the diode is switched from closed $(U=10 \mathrm{~V})$ to open mode $(U=-2 \mathrm{~V}), t_{t r}=0.1 \mathrm{~s}$. As a result of increasing $p$, the local maximum (overshoot) disappears.

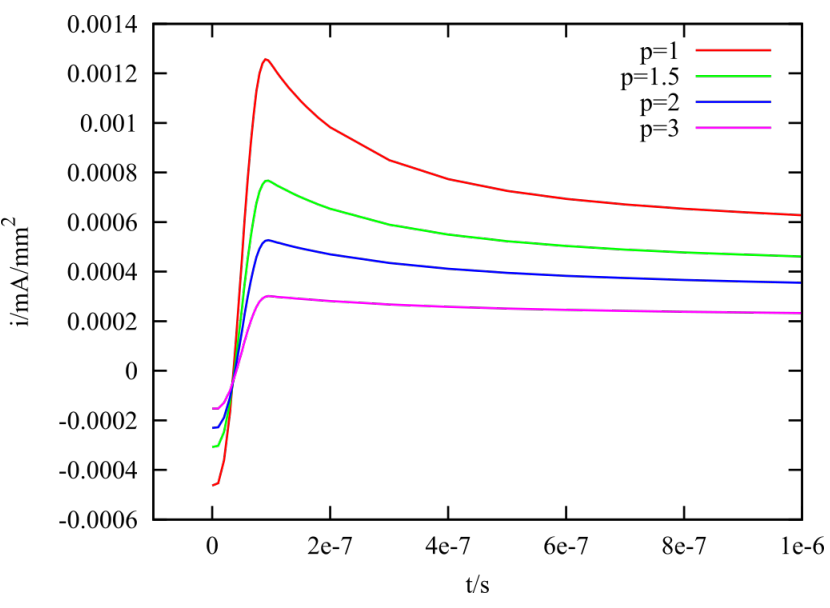

Fig. 11 Simulated time - current density curve of the diode (magnified between $t=0$ to $t=1 \mathrm{e}-6 \mathrm{~s}$ ) in case of different $p$ values, if the diode is switched from closed $(U=10 \mathrm{~V})$ to open mode $(U=-2 \mathrm{~V}), t_{t r}=1 \mathrm{e}-7 \mathrm{~s}$.

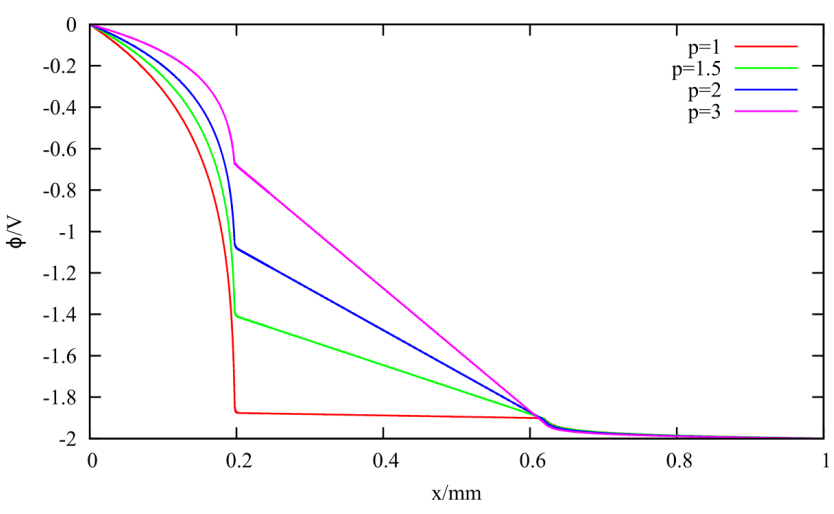

Fig. 12 Simulated potential profiles belonging to the overshoot of the diode in case of different $p$ values, $t_{t r}=1 \mathrm{e}-7 \mathrm{~s}$.

\section{Conclusion}

Our main aim was to study by model calculations the time response of the acid-base diode for polarity change (when the diode is switched from closed to open mode). In the simulation study various hypothetic connecting elements differing in the diffusion coefficients of hydrogen and hydroxide ions were modeled. We found that these diffusion coefficients have an impact on the time - current density curves, depending on the transition time $\left(t_{t r}\right)$ an overshoot (local maximum followed by a local minimum) on the time - current density curve can exist. The bigger the diffusion coefficients are, the more pronounced the overshoot becomes. This is in agreement with the experiments, where overshoot was found in PVA hydrogels, but not in PVB membranes.

\section{Acknowledgement}

This work was partially supported by OTKA Grant K131425. 


\section{References}

[1] Noszticzius, Z., Schubert, A. "Electrolyte Diode I. Analysis of Isothermal Transport Processes in the Interfaces of Aqueous Solution of Acids and Bases. I", Periodica Polytechnica Chemical Engineering, 17(2), pp. 165-177, 1973. [online] Available at: https:// pp.bme.hu/ch/article/view/3226 [Accessed: 06 August 2020]

[2] Schubert, A., Noszticzius, Z. "Electrolyte Diode-An Experimental Study. Polarization Phenomena at the Junction of the Aqueous Solutions of an Acid and a Base. Part II", Periodica Polytechnica Mechanical Engineering, 21(3-4), pp. 279-283, 1977. [online] Available at: https://pp.bme.hu/me/article/view/5928 [Accessed: 06 August 2020]

[3] Hegedűs, L., Noszticzius, Z., Papp, Á., Schubert, A., Wittmann, M. "Polarization phenomena in hydrogel membranes. Experimental realization of an electrolyte diode", Ach-models in Chemistry, 132, pp. 207-224, 1995.

[4] Koncz, V., Noszticzius, Z., Roszol, L., Kály-Kullai, K. "TimeDependent Modeling of Salt-Contaminated Acid-Base Diodes", Journal of The Electrochemical Society, 164(4), pp. H257-H265, 2017.

https://doi.org/10.1149/2.1701704jes

[5] Roszol, L., Várnai, A., Lorántfy, B., Noszticzius, Z., Wittmann, M. "Negative salt effect in an acid-base diode: Simulations and experiments", The Journal of Chemical Physics, 132(6), Article number: $064902,2010$. https://doi.org/10.1063/1.3292001

[6] Iván, K., Kirschner, N., Wittmann, M., Simon, P. L., Jakab, V., Noszticzius, Z., Merkin, J. H., Scott, S. K. "Direct evidence for fixed ionic groups in the hydrogel of an electrolyte diode", Physical Chemistry Chemical Physics, 4(8), pp. 1339-1347, 2002. https://doi.org/10.1039/B109016J

[7] Iván, K., Wittmann, M., Simon, P. L., Noszticzius, Z., Vollmer, J. "Electrolyte diodes and hydrogels: Determination of concentration and $p \mathrm{~K}$ value of fixed acidic groups in a weakly charged hydrogel", Physical Review E, 70(6), Article number: 061402, 2004. https://oi.org/10.1103/PhysRevE.70.061402

[8] Roszol, L., Lawson, T., Koncz, V., Noszticzius, Z., Wittmann, M., Sarkadi, T., Koppa, P. "Micropatterned Polyvinyl Butyral Membrane for Acid-Base Diodes", Journal of Physical Chemistry B, 114(43), pp. 13718-13725, 2010. https://doi.org/10.1021/jp106773y
[9] Slouka, Z., Přibyl, M., Šnita, D., Postler, T. "Transient behavior of an electrolyte diode", Physical Chemistry Chemical Physics, 9(39), pp. 5374-5381, 2007. https://doi.org/10.1039/B707197C

[10] Přibyl, M., Šnita, D., Kubíček, M. "Adaptive mesh simulations of ionic systems in microcapillaries based on the estimation of transport times", Computers \& Chemical Engineering, 30(4), pp. 674-685, 2006. https://doi.org/10.1016/j.compchemeng.2005.11.006

[11] Hegedüs, L., Wittmann, M., Kirschner, N., Noszticzius, Z. "Reaction, diffusion, electric conduction and determination of fixed ions in a hydrogel", In: Zrínyi, M. (ed.) Gels. Progress in Colloid \& Polymer Science, Steinkopff, Heidelberg, Germany, 1996, pp. 101-109. https://doi.org/10.1007/BFb0114397

[12] Merkin, J. H., Simon, P. L., Noszticzius, Z. "Analysis of the electrolyte diode. Electro-diffusion and chemical reaction within a hydrogel reactor", Journal of Mathematical Chemistry, 28(1), pp. 43-58, 2000. https://doi.org/10.1023/A:1018840309923

[13] Lindner, J., Šnita, D., Marek, M. "Modelling of ionic systems with a narrow acid-base boundary", Physical Chemistry Chemical Physics, 4(8), pp. 1348-1354, 2002. https://doi.org/10.1039/B109525K

[14] Šnita, D., Pačes, M., Lindner, J., Kosek, J., Marek, M. "Nonlinear behavior of simple ionic systems in hydrogel in an electric field", Faraday Discussion, 120, pp. 53-66, 2002. https://doi.org/10.1039/B103530B

[15] Iván, K., Simon, P. L., Wittmann, M., Noszticzius, Z. "Electrolyte diodes with weak acids and bases. I. Theory and an approximate analytical solution", The Journal of Chemical Physics, 123(16), Article number: 164509, 2005. https://doi.org/10.1063/1.2085047

[16] Iván, K., Wittmann, M., Simon, P. L., Noszticzius, Z. "Electrolyte diodes with weak acids and bases. II. Numerical model calculations and experiments", The Journal of Chemical Physics, 123(16), Article number: 164510, 2005. https://doi.org/10.1063/1.2085049

[17] Marcus, Y. "Ion properties", Marcel Dekker, New York, NY, USA, 1997. 


\section{Appendix}

A couple of years ago a new connecting element for the diode was developed: a PVB membrane [8]. During the measurement of the characteristic of the diode, when voltage was switched, overshoots were observed in the case of PVA gel cylinder and were not observed in the case of PVB membrane (see Fig. 13). The transients were investigated by switching the voltage between forward $(0.2 \mathrm{~V})$ and reverse ( $-2 \mathrm{~V})$ mode periodically (see Fig. 13). Overshoots appeared when switching from forward to reverse mode. In the case of PVA gel cylinder a similar rapid response of the diode with overshoots was observed by Slouka et al. [9], when the diode was switched from $0 \mathrm{~V}$ to $10 \mathrm{~V}$ or $-10 \mathrm{~V}$.

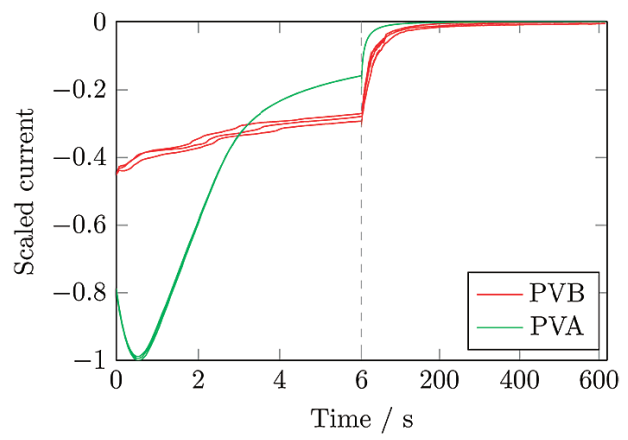

Fig. 13 Scaled transient current after the voltage is switched from forward $(0.2 \mathrm{~V})$ to reverse $(-2 \mathrm{~V})$ mode, three periods are shown together. The period time is about $20.5 \mathrm{~min}$. The concentrations in the reservoirs are: $[\mathrm{KOH}]=[\mathrm{HCl}]=0.1 \mathrm{M}$. For this figure three periods are shifted to a common starting point, and the currents are scaled. First, the current of PVB is scaled to give the same steady-state current in forward mode as the PVA, then both currents are normalized with the same factor to give a minimum scaled current of -1 for PVA. This figure was originally published in [8]. 